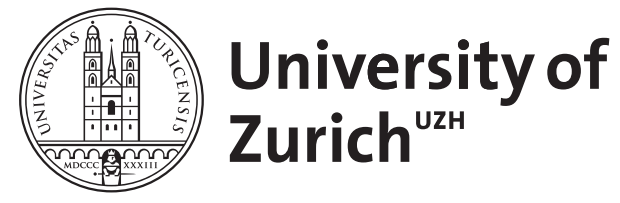

\title{
Les occlusives palatales du Vallader
}

\author{
Schmid, Stephan
}

Posted at the Zurich Open Repository and Archive, University of Zurich ZORA URL: https://doi.org/10.5167/uzh-45248

Book Section

Originally published at:

Schmid, Stephan (2010). Les occlusives palatales du Vallader. In: Iliescu, M; Siller-Runggaldier, H; Danler, P. Actes du XXVe Congrès International de Linguistique et de Philologie Romanes. Tome II. Berlin - New York: De Gruyter, 185-194. 


\section{Stephan Schmid}

\section{Les occlusives palatales du vallader}

\section{Introduction}

La présente contribution porte sur la nature phonétique d'un type particulier de consonnes du romanche parlé en Basse-Engadine (Suisse), à savoir les occlusives palatales $[\mathrm{c} f]$. Après une brève caractérisation sociolinguistique du vallader, nous envisagerons ces deux consonnes du point de vue diachronique, géolinguistique et typologique, en posant aussi le problème de leur transcription phonétique. La partie principale de notre étude consiste en la présentation d'une recherche empirique qui vise à saisir la nature articulatoire de ces consonnes avec les outils de la phonétique acoustique.

Afin de distinguer [ $\mathrm{c} f$ ] par rapport au lieu et au mode d'articulation de leurs consonnes 〈voisines〉 [ $\left.\mathrm{t} \int \mathrm{d}_{3}\right]$ et $[\mathrm{k} \mathrm{g}]$, un corpus de mots lus par trois locutrices natives du vallader a été constitué au laboratoire de phonétique de l'Université de Zurich. Des mesures et calculs ont été effectués quant à la dimension temporelle et spectrale des sons obtenus. Selon les résultats de l'analyse acoustique, la différentiation entre occlusives palatales et affriquées postalvéolaires semble être moins nette que ce à quoi nous nous étions attendus, ce qui pourrait amener les locuteurs du vallader à abandonner dans le futur la distinction entre ces deux catégories.

\section{Le vallader}

Selon l'article 4 de la Constitution fédérale, le romanche est la quatrième langue nationale de la Confédération suisse. Néanmoins, le romanche peut être considéré comme une langue (virtuelle〉 (Hilty à paraître). Il existe bien une tradition scripturale séculaire documentée pour chacun des cinq idiomes rhéto-romans parlés dans le canton des Grisons (à savoir le sursilvan dans la région du Rhin antérieur, le sutsilvan dans la région du Rhin postérieur, le surmiran dans les vallées de l'Albula et du Julier, le puter en Haute-Engadine et le vallader en Basse-Engadine et dans la Val Müstair), mais la tentative d'introduire une norme standard unifiée est relativement récente.

Dans le recensement fédéral de la population réalisé en l'an 2000, 35095 personnes se sont déclarées locuteurs natifs du romanche, ce qui correspond à $0,5 \%$ de la population suisse (Lüdi / Werlen 2005: 7); seules 5138 personnes ont indiqué le vallader comme langue principale (Gross 2004: 31). Du point de vue sociolinguistique, on peut donc 
qualifier le vallader de 〈langue menacée`, d'autant plus que tous les locuteurs du romanche parlent aussi le suisse allemand.

\section{Les occlusives palatales}

\subsection{Le système consonantique du vallader}

Le système phonologique du vallader compte 27 phonèmes consonantiques (Schmid 2007: 33):

\begin{tabular}{lcccccc}
\hline & Labial & Alvéolaire & $\begin{array}{c}\text { Post- } \\
\text { alvéolaire }\end{array}$ & Palatal & Vélaire & Glottal \\
\hline Occlusive & $\mathrm{p} \mathrm{b}$ & $\mathrm{t} \mathrm{d}$ & & $\mathrm{c} \mathrm{j}$ & $\mathrm{k} \mathrm{g}$ & \\
Fricative & $\mathrm{f} \mathrm{v}$ & $\mathrm{s} \mathrm{z}$ & $\int 3$ & & & $\mathrm{~h}$ \\
Affriquée & & $\mathrm{ts} \mathrm{dz}$ & $\mathrm{t} \int \mathrm{d} 3$ & & & \\
Nasale & $\mathrm{m}$ & $\mathrm{n}$ & & $\mathrm{j}$ & & \\
Vibrante & & $\mathrm{r}$ & & & & \\
Latérale & & $\mathrm{l}$ & & $\kappa$ & & \\
Approximante & & & $\mathrm{j}$ & $\mathrm{w}$ & \\
\hline
\end{tabular}

Tableau 1: Phonèmes consonantiques du vallader

Il s'agit d'un système assez 〈économique〉 et typologiquement non marqué, avec la seule exception de la région palatale, où l'on trouve deux consonnes relativement rares dans les langues du monde, à savoir les occlusives $/ \mathrm{c} \mathrm{j} /$. En outre, du point de vue articulatoire et perceptif ces consonnes sont très proches des affriquées postalvéolaires $/ \mathrm{t} \int \mathrm{d} z /$.

Néanmoins, il faut noter la grande fréquence de ces consonnes dans le lexique du vallader. Il est vrai qu'on ne trouve pas beaucoup de paires minimales qui exploitent l'opposition entre /c/ et $/ \mathrm{t} \mathrm{f} /$, comme par exemple chatta «(elle) trouve〉 vs. tschatta ((la) patte>; toutefois, les occlusives palatales apparaissent dans divers contextes phonotactiques, non seulement à l'initiale du mot (1a.) et entre voyelles (1b.), mais aussi après consonne (1c.) et à la finale du mot (1d.).
(1) a. chan «chien〉 giat «chat〉
b. vacha 〈vache〉 magöl 〈verre〉
c. grazcha 〈merci〉 gövgia $\langle$ jeudi $>$
d. fich «beaucoup〉 bös-ch «arbre〉

Il est donc non seulement difficile de distinguer entre occlusives palatales et affriquées postalvéolaires, mais il est également difficile de prononcer ces groupes de consonnes. C'est pourquoi on les trouve souvent dans des proverbes (2a.) et dans des virelangues (2b.). 
(2) a. Chi chi tschercha, chatta «Celui qui cherche, trouve〉.

b. I I 'eira ün chatschader sün üna tschücha chi vaiva tschüf tschinch chamuotschs ¿Il y avait un chasseur sur une souche qui avait attrapé cinq chamoix».

3.2 Origine diachronique et diffusion diatopique des occlusives palatales

L'origine diachronique des occlusives palatales est due à divers processus phonologiques:

\begin{tabular}{|c|c|c|c|c|c|c|}
\hline & K- & & G- & & J- & \\
\hline $\begin{array}{l}-\mathrm{A} \\
-\overline{\mathrm{U}}\end{array}$ & & $\begin{array}{l}\text { chan 〈chien〉 } \\
\text { chüna 〈berceau〉 }\end{array}$ & {$[\mathrm{f}]$} & giat «chat〉 & {$[\mathrm{f}]$} & $\begin{array}{l}\text { giantar 〈déjeuner〉 } \\
\text { gün 〈juin〉 }\end{array}$ \\
\hline $\begin{array}{l}-\mathrm{O} \\
-\breve{U}\end{array}$ & {$[\mathrm{k}]$} & $\begin{array}{l}\text { corp 〈corps〉 } \\
\text { cuort }\langle\text { court }\rangle\end{array}$ & [g] & gust 〈goût〉 & {$[\mathrm{f}]$} & $\begin{array}{l}\text { gö 〈jeu〉 } \\
\text { giuven 〈jeune〉 }\end{array}$ \\
\hline $\begin{array}{l}-E \\
-I\end{array}$ & {$\left[\mathrm{t} \int\right]$} & $\begin{array}{l}t s c h e ̂ l 〈 c i e l 〉 \\
\text { tschinch 〈cinq〉 }\end{array}$ & [d3] & dschender (gendre) & & \\
\hline
\end{tabular}

Tableau 2: Origine diachronique des obstruentes palatales du vallader

Le lexique connaît de nombreuses exceptions (liées en partie au traitement des latinismes et des italianismes), mais il est clair que le processus phonologique le plus productif est la palatalisation de K,G devant la voyelle $\mathrm{A}$. En outre, on repère les occlusives palatales devant la voyelle $<\ddot{\mathrm{u}}>$ et comme résultat de yod.

La palatalisation des occlusives vélaires latines devant $\mathrm{A}$ est considérée comme un trait caractéristique des parlers 〈rhéto-romans〉 dans le sens vaste du terme, autrement dit non seulement du romanche des Grisons, mais aussi du ladin dolomitique et du frioulan. Cependant, la diffusion aréale des occlusives palatales est plus vaste et couvre aussi quelques dialectes gallo-italiques situés dans l'arc alpin, par exemple dans le Canton du Tessin ou même dans le Piémont, plus précisément dans la Valsesia (Berruto 1974: 30; Romano et al. 2005). En effet, comme l'a montré le romaniste zurichois Heinrich Schmid (1956: 53-80), la palatalisation de K,G devant la voyelle A constitue une innovation par rapport au latin et devait être répandue dans toute l'Italie septentrionale (cf. aussi Vigolo 1986; Videsott 2001: 28-30), mais elle représente aujourd'hui un trait conservateur, typique des régions latérales.

\subsection{Considérations typologiques}

Les occlusives palatales ne sont pas seulement marginales à l'intérieur des langues romanes, elles sont aussi typologiquement marquées. Ainsi, si l'on consulte la base de données UPSID qui recueille les inventaires des phonèmes de 451 langues représentatives du point de vue des familles génétiques (Maddieson 1984; Maddieson / Precoda 1991), on constate que les occlusives palatales sont beaucoup moins fréquentes que les affriquées postalvéolaires, qui à leur tour sont moins fréquentes que les occlusives vélaires. 


\begin{tabular}{|c|c|c|c|c|c|}
\hline $\mathrm{c}$ & $\mathrm{f}$ & $\mathrm{t} \int$ & $\mathrm{d} 3$ & $\mathrm{k}$ & $\mathrm{g}$ \\
\hline $11.97 \%$ & $09.53 \%$ & $41.69 \%$ & $25.06 \%$ & $89.36 \%$ & $56.10 \%$ \\
\hline
\end{tabular}

Tableau 3: Fréquence des obstruentes palatales et vélaires selon UPSID

Si le vallader est une variété de langue en danger par son statut sociolinguistique, les occlusives palatales pourraient être menacées à leur tour par la position qu'elles occupent dans le système linguistique. En effet, dans la partie orientale de la Basse-Engadine, le phonème sonore $/ \mathfrak{y} /$ est en train d'être abandonné en faveur de l'approximante $/ \mathrm{j} /$. Ce n'est pas par hasard que ces consonnes ont disparu dans de nombreux parlers de l'Italie du Nord qui les possédaient autrefois (cf. 5.). Avant de nous interroger sur le destin de ces sons en vallader, nous allons aborder le problème de leur description phonétique.

\subsection{La caractérisation phonétique des obstruentes palatales}

Il faut dire que la caractérisation des obstruentes palatales ne fait pas l'unanimité des romanistes qui se sont penchés sur le vallader. Les descriptions diffèrent par rapport au mode d'articulation, au lieu d'articulation et aux symboles API choisis pour la transcription phonétique des ces consonnes. Or, si quelques-uns les traitent comme des occlusives palatales, d'autres les considèrent comme des affriquées alvéolo-palatales. Par conséquent, on adopte des choix différents dans la transcription phonétique: par exemple, les manuels de Haiman / Benincà (1992: 29, 33) et de Liver (1999: 64) emploient pour le vallader les symboles des occlusives palatales [ $\mathrm{c} \mathfrak{j}$ ], tandis que dans le dictionnaire français-vallader de Gilbert Taggart (1990: 11) on trouve les symboles des affriquées alvéolo-palatales [tc dz] typiques du polonais (Jassem 2003: 103). ${ }^{1}$

Les consonnes palatales du romanche figuraient sur la liste des «sons difficiles〉 des dialectes suisses qui faisaient l'objet d'un programme de recherche inauguré par Eugen Dieth, fondateur du laboratoire de phonétique de l'Université de Zurich. Les résultats d'une enquête expérimentale menée chez huit locuteurs provenant de diverses localités des Grisons montrent non seulement des différences entre deux types d'articulation, mais aussi des différences entre les dialectes du romanche. En effet, sur la base de l'observation de palatogrammes, Brunner (1963) distingue une affriquée postalvéolaire d'une consonne non affriquée et articulée avec plus de contact dans la région palatale, ce qui serait plus évident dans le sursilvan que dans les autres variétés de romanche.

À plus de quarante ans de distance, la présente étude reprend le programme de recherche de Dieth et Brunner en y appliquant une démarche acoustique plutôt qu'articulatoire. Dans le paragraphe suivant, nous présenterons les premiers résultats de notre enquête empirique.

1 Notons que de telles divergences se retrouvent aussi dans la description d'autres langues: par exemple, les obstruentes palatales du hongrois sont caractérisées comme des affriquées par Szende (1999: 104), tandis que Sziptár / Törkenczy (2000: 82-83) les considèrent comme des occlusives. 


\section{4. Étude acoustique}

\subsection{Données et méthode}

Les données qui nous ont permis de mener la présente enquête ont été recueillies grâce à la collaboration de trois locutrices natives de vallader (MN, MP, MD), âgées d'environ 30, 50 et 70 ans. Les locutrices ont été enregistrées dans une maison privée et dans des bureaux de notre laboratoire avec un enregistreur portable Marantz PDM 671 sur carte flash et avec un microphone Sennheiser ME66 (directivité supercardioïde/lobe, réponse en fréquence 50$20000 \mathrm{~Hz} \pm 2,5 \mathrm{~dB}$ ). Les enregistrements, réalisés avec une fréquence d'échantillonage de $44.1 \mathrm{kHz}$ et une quantisation de 16 bit, ont été sauvés sur ordinateur comme fichiers sonores avec une extension «.wav».

En ce qui concerne la structure linguistique du corpus, les trois locutrices ont lu 45 syntagmes qui contiennent les consonnes / $\mathrm{cf} t \int \mathrm{d}_{3} \mathrm{k} \mathrm{g} \mathrm{j} /$ à l'initiale du mot. Pour faciliter la segmentation, les mots ont été placés à l'intérieur de syntagmes du type ella tschüffa, la giuvna, il chucal (autrement dit après /a/ et /1/ et avant une voyelle tonique). En outre, pour chaque consonne, on a cherché des séries de mots qui contiennent sept voyelles différentes (par exemple chasa, checla, chicra, chombra, chucal, chüna, chöntcha) afin de neutraliser une éventuelle influence coarticulatoire du contexte vocalique sur les propriétés spectrales de la consonne en question. Finalement, les syntagmes ont été lus à l'intérieur de trois phrases cadre Eu n’ha dit _ la prüma, seguonda, terza jada (‘j’ai dit _ pour la première, deuxième, troisième fois ), afin de réduire une éventuelle influence du contexte prosodique sur la durée des sons en question.

Pour l'analyse acoustique, effectuée à l'aide du logiciel Praat (Boersma / Weenink 2008), nous avons tenu compte des paramètres de durée, d'intensité, et de certaines propriétes spectrales liées aux timbres des différentes consonnes (cf. 4.4).

\subsection{Durée}

Pour l'analyse de la dimension temporelle des occlusives et des affriquées, il faut considérer non seulement la durée totale du segment, mais aussi le rapport entre la phase de relâchement et cette durée totale.

L'histogramme de la figure 1 montre les moyennes arithmétiques pour toutes les réalisations produites par les trois locutrices. On constate qu'en général les consonnes sourdes sont plus longues que les sonores. Quant au lieu d'articulation, les durées moyennes sont de $159 \mathrm{~ms}$ pour [t $\mathrm{t}$ ], de $157 \mathrm{~ms}$ pour [c] et de $136 \mathrm{~ms}$ pour [k]; l'occlusive palatale est donc beaucoup plus proche de l'affriquée postalvéolaire que de l'occlusive vélaire. Quant au rapport entre la phase de relâchement et la durée totale du segment, la consonne palatale dépasse de $48 \%$ la consonne postalvéolaire, dans laquelle la phase de tenue remonte à $46 \%$ de la durée totale. En tous les cas, les palatales montrent un rapport de durée entre les deux phases presque identique à celui des postalvéolaires et se distancient clairement des vélaires. Sur la base de ces données temporelles, on peut ainsi affirmer que les obstruentes palatales du vallader se rapprochent plus des affriquées que des occlusives. 


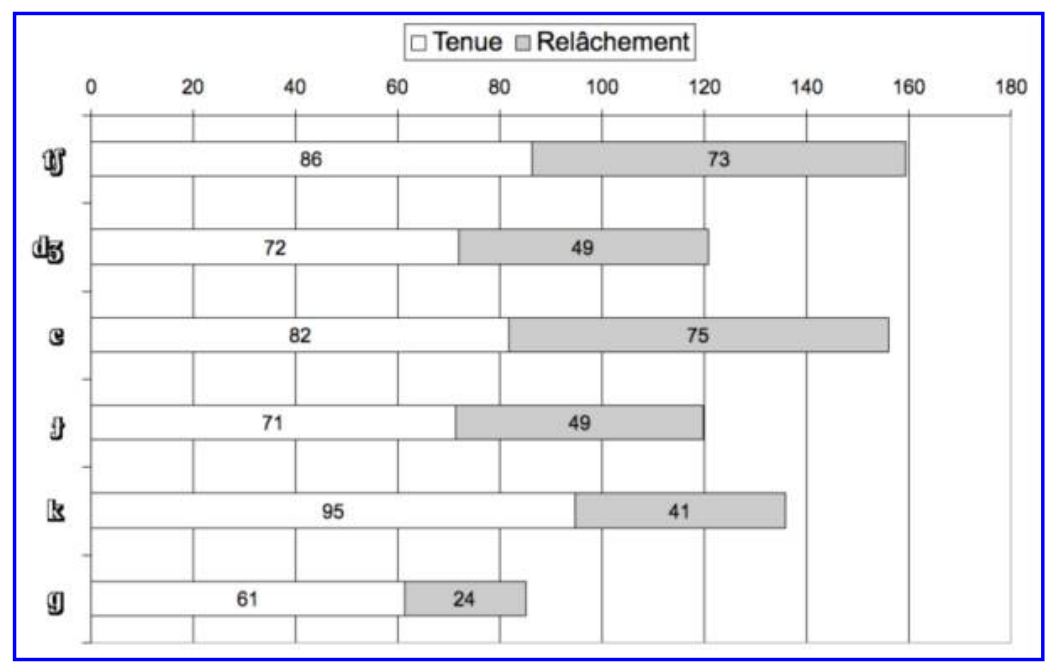

Figure 1: Durées moyennes des trois locutrices (ms)

Cette impression est confirmée si nous comparons les trois locutrices par rapport au pourcentage du relâchement sur la durée totale. L'histogramme en figure 2 montre que les trois locutrices se comportent de façon constante, puisque la plus jeune (MN) présente toujours les pourcentages les plus hauts. En effet, elle est la seule à produire des relâchements relativement longs dans les consonnes postalvéolaires, tandis que les autres locutrices présentent un pourcentage plus élevé dans les palatales. La séparation des données selon les trois locutrices confirme dès lors les considérations faites sur la base des moyennes du corpus entier.

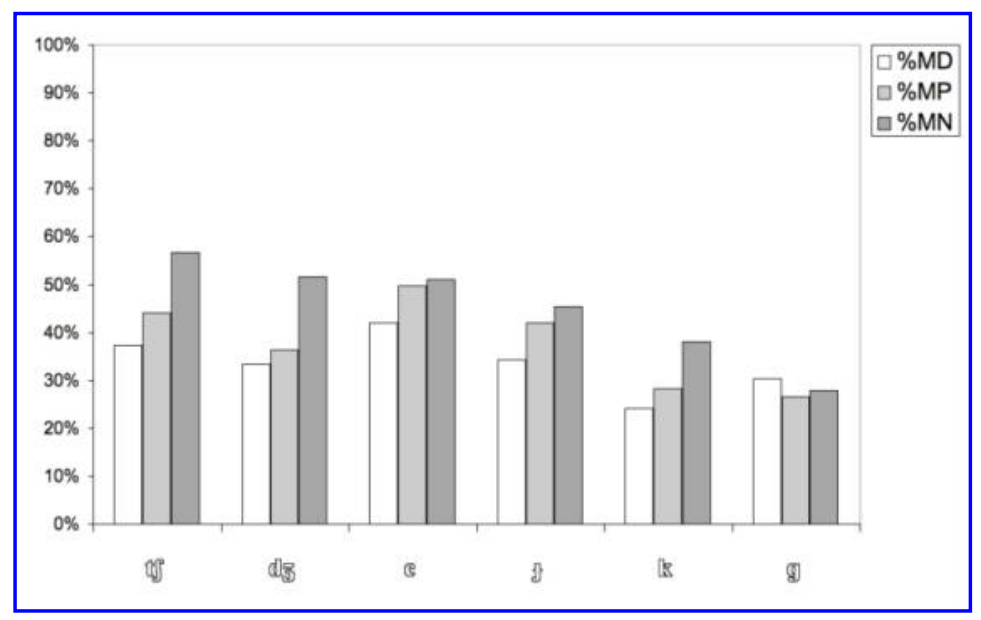

Figure 2: Pourcentage du relâchement sur la durée totale (comparaison entre les trois locutrices)

Nous allons à présent laisser de côté les occlusives vélaires, pour nous concentrer sur la différence entre consonnes palatales et postalvéolaires. 


\subsection{Intensité}

Dans les langues du monde, certaines fricatives présentent un volume plus haut que d'autres: par exemple, les sibilantes produisent un bruit plus fort que les labiodentales et les interdentales. Sur la base de l'impression auditive, on pourrait postuler que les postalvéolaires sont articulées avec une friction plus intense des palatales.

\begin{tabular}{|c|c|c|c|c|}
\hline & MD & MP & MN & Moyenne \\
\hline c & 62.7 & 61.1 & 58.9 & 60.9 \\
\hline J & 63.0 & 62.1 & 57.1 & 60.7 \\
\hline t & 62.4 & 62.1 & 60.8 & 61.8 \\
\hline d3 & 62.0 & 65.2 & 58.7 & 62.0 \\
\hline
\end{tabular}

Tableau 4: Intensité moyenne dans la phase de relâchement (dB)

Cependant, le tableau 4 ne montre aucune différence significative par rapport à l'intensité de ces deux catégories de sons. En mesurant l'énergie moyenne dans la phase de relâchement, on obtient des valeurs presque égales pour les deux lieux d'articulation, avec environ un seul décibel de plus sur soixante pour les postalvéolaires. De plus, dans le cas de la première locutrice, ce sont les palatales qui présentent une intensité légèrement plus élevée. Pour l'instant, l'énergie globale du bruit de friction ne semble guère permettre de distinguer les palatales des postalvéolaires.

\subsection{Timbre}

Il faut remarquer que l'analyse acoustique des consonnes non voisées est beaucoup plus ardue que celle des voyelles, par exemple, à cause de la nature apériodique du signal. On trouve en effet une grande variabilité dans le spectre acoustique, qui peut changer de forme à tout instant. C'est pourquoi, notamment dans l'analyse acoustique des fricatives, on recourt souvent à la notion de centre de gravité proposée par Forrest et al. (1988) et appliquée avec succès dans une étude comparée des fricatives de sept langues différentes (Gordon et al. 2002).

Le centre de gravité est une espèce de centroïde spectral obtenu en multipliant toutes les valeurs de fréquence dans le spectre numérique par les valeurs respectives d'intensité, pour ensuite diviser la somme de ces produits par la somme de toutes les valeurs de fréquence du spectre. Le centre de gravité est considéré comme un témoin de la brillance d'un son; il est corrélé avec la grandeur de la cavité antérieure de la bouche, dans le sens où sa valeur augmente pour les articulations antérieures, tandis qu'elle diminue pour les articulations postérieures.

Or, le tableau 5 montre que même cette méthode ne permet pas de distinguer clairement les palatales et les postalvéolaires en vallader, vu que les moyennes pour les trois locutrices se situent toutes entre $4600 \mathrm{~Hz}$ et $5000 \mathrm{~Hz}$. Le centre de gravité des palatales est légèrement plus haut, ce qui confirme l'impression d'un timbre plus clair. D'un point de vue articulatoire, on peut interpréter ce résultat en affirmant que la cavité de résonance des 
postalvéolaires est plus longue à cause de l'avancement des lèvres, tandis que les palatales seraient produites avec une position écartée des lèvres.

\begin{tabular}{|c|c|c|c|c|}
\hline & MD & MP & MN & Moyenne \\
\hline c & 4303 & 5579 & 5012 & 4965 \\
\hline J & 3882 & 6089 & 5005 & 4992 \\
\hline t & 4050 & 5402 & 5112 & 4855 \\
\hline d3 & 3589 & 6032 & 4325 & 4649 \\
\hline
\end{tabular}

Tableau 5: Centre de gravité $(\mathrm{Hz})$

Mais on note aussi une très grande variabilité entre les trois locutrices. En général, les valeurs de MD sont constamment plus basses alors que celles de MP sont constamment plus hautes. En outre, dans le cas de MN, le centre de gravité de l'affriquée postalvéolaire dépasse celui de la consonne palatale, en dépit de la tendance générale.

Quelles conclusions peut-on donc tirer de ces résultats préliminaires? Le manque de différenciation entre les deux catégories est-il le reflet de la substance physique des sons, ou est-il plutôt dû à la nature approximative de la méthode du centre de gravité? Pour pouvoir répondre à ces questions, d'autres analyses complémentaires seront nécessaires. Sur le plan acoustique, l'examen des enveloppes spectrales peut ajouter des informations sur le caractère plus ou moins compact ou diffus des sons. De plus, l'inspection des transitions des formants à travers l'équation des loci a été appliquée avec succès par Romano et al. (2005) aux occlusives palatales du dialecte de la Valsesia.

\section{Conclusions}

Dans l'état actuel de la recherche, il n'est pas possible de se décider de façon catégorique en faveur de l'une ou de l'autre transcription qui ont été proposées pour les obstruentes palatales. Si l'on adopte une vision large de la palatalité, comme celle de Keating / Lahiri (1993: 79-81, 95) dans leur analyse du tchèque, les symboles des occlusives restent plausibles. Si l'on veut insister sur la nature d'affriquée de ces consonnes, on peut les transcrire 〈à la polonaise), même si la prépondérance de l'élément alvéolaire est encore à démontrer.

Mais il est aussi certain que les deux catégories de consonnes sont susceptibles d'être confondues. Déjà Weinreich (1953: §2.2) observait que les germanophones de la Tumliastga réinterprétaient les occlusives palatales du sutsilvan comme des affriquées postalvéolaires. Au début de notre contribution, nous avons mentionné le fait que les occlusives palatales devaient être plus répandues dans l'Italie du Nord. Ainsi, Politzer (1967: 49) a montré que dans certains parlers de la Val di Non les occlusives palatales ont été remplacées par les affriquées postalvéolaires dans le courant du vingtième siècle. Nous ne pouvons pas exclure que tel sera le destin du vallader. 


\section{Bibliographie}

Berruto, Gaetano (1974): Piemonte (Profilo dei dialetti italiani 1). Pisa: Pacini.

Boersma, Paul / Weenink, David (2008): Praat: doing phonetics by computer. www.praat.org.

Brunner, Rudolf (1963): Zur Physiologie der rätoromanischen Affrikaten tsch und $\mathrm{tg}$ (ch): ein Beitrag zur Kenntnis von palatalen und palatalisierten Artikulationen. In: Zinsli, Paul et al. (edd.): Sprachleben der Schweiz. Sprachwissenschaft, Namenforschung, Volkskunde. Bern: Francke, 167173.

Forrest, Karen / Weismer, Gary / Milenkovic, Paul / Dougall, Ronald N. (1988): Statistical analysis of word-initial obstruents: preliminary data. In: Journal of the Acoustical Society of America 84, 115-123.

Gordon, Matthew / Barthmaier, Paul / Sands, Kathy (2002): A cross-linguistic acoustic study of voiceless fricatives. In: Journal of the International Phonetic Association 32, 141-174.

Gross, Manfred (2004): Rumantsch. Facts \& Figures. Coire: Lia Rumantscha.

Haiman, John / Benincà, Paola (1992): The Rheto-romance languages. London / New York: Routledge.

Hilty, Gerold (à paraître): La storia del romancio e la questione ladina. In: ACILPR XXV.

Jassem, Wiktor (2003): Polish. In: Journal of the International Phonetic Association 33, 103-107.

Keating, Patricia / Lahiri, Aditi (1993): Fronted velars, palatalized velars, and palatals. In: Phonetica 50, 73-101.

Liver, Ricarda (1999): Rätoromanisch. Eine Einführung in das Bündnerromanische. Tübingen: Narr.

Lüdi, Georges / Werlen, Iwar (2005): Le paysage linguistique en Suisse. Neuchâtel: Office fédéral de la statistique.

Maddieson, Ian (1984): Patterns of sounds. Cambridge: Cambridge University Press.

- / Precoda, Kristin (1991): Updating UPSID. In: UCLA Working Papers in Phonetics 74, 104-114.

Politzer, Robert L. (1967): Beiträge zur Phonologie der Nonsberger Mundart. Bregenz: Ruß.

Romano, Antonio / Molino, Gianni / Rivoira, Matteo (2005): Caratteristiche acustiche e articolatorie delle occlusive palatali: alcuni esempi del Piemonte e di altre aree italo-romanze. In: Cosi, Piero (ed.): Misura dei parametri. Aspetti tecnologici ed implicazioni nei modelli linguistici. Torriana: EDK Editore, 389-428.

Schmid, Heinrich (1956): Über Randgebiete und Sprachgrenzen. In: VR 15, 19-80.

Schmid, Stephan (2007): Einführung in die allgemeine Phonetik und Phonologie, für Studierende der Romanistik. Materialien. Zürich: Phonetisches Laboratorium der Universität Zürich.

Szende, Tamás (1999): Hungarian. In: Handbook of the International Phonetic Association. Cambridge: Cambridge University Press, 104-107.

Sziptár, Péter / Törkenczy, Miklós (2000): The Phonology of Hungarian. Oxford: Oxford University Press.

Taggart, Gilbert (1990): Dictionnaire du vocabulaire fondamental: romanche vallader - français et français - romanche vallader. Coire: Lia Rumantscha.

Videsott, Paul (2001): La palatalizzazione di CA e GA nell'arco alpino orientale. Un contributo alla delimitazione dei confini dell'Italia linguistica nell'anno 1000. In: VR 60, 25-50.

Vigolo, Maria Teresa (1986): La palatalizzazione di $\mathrm{C}, \mathrm{G}^{+\mathrm{a}}$ nei dialetti veneti. In: AGI 71, 60-80.

Weinreich, Uriel (1953): Languages in contact: findings and problems. New York: Linguistic Cercle of New York (n. 1). 
